\title{
An Explicit Form of Heaviside Step Function
}

J. Venetis

Section of Mechanics, Faculty of Applied Mathematics and Physical Sciences, National Technical University of Athens Email: johnvenetis4@gmail.com

\begin{abstract}
In this paper, the author derives an explicit form of Heaviside Step Function, which evidently constitutes a fundamental concept of Operational Calculus and is also involved in many other fields of applied and engineering mathematics.

In particular, this special function is exhibited in a very simple manner as a summation of four inverse tangent functions. The novelty of this work is that the proposed exact formulae are not performed in terms of miscellaneous special functions, e.g. Bessel functions, Error function, Beta function etc and also are neither the limit of a function, nor the limit of a sequence of functions with point - wise or uniform convergence.

Hence, this formula may be much more appropriate and useful in the computational procedures which are inserted into Operational Calculus techniques and other engineering practices.
\end{abstract}

\section{Keywords}

Heaviside function, explicit form, inverse trigonometric functions

\section{Introduction}

The Heaviside step function, or unit step function, which is usually notated by the symbols $\mathrm{H}$ or $\mathrm{u}$, is a discontinuous single - valued function, the value of which is zero for negative argument and equal to unity for positive argument [1]. This special function was introduced by Oliver Heaviside, who was an important pioneer in the study of electronics and also made a remarkable contribution to the field of Operational Calculus [2]. A very significunt property of this function is that it is capable of being represented either as a piecewise constant function or as a generalized function [1,3]. The unit step function is mainly used in the calculation processes of Control Theory and signal processing in order to represent a signal which switches on at a specified time and stays switched on indefinitely. This function is also implemented together with its derivative, i.e. Dirac delta function in structural engineering in order to describe various types of structural loads, e.g. off - axis four point bending of simply supported or fully constrained beams. Hence, it is very useful for the necessary calculations dealing with conceptual and embodiment design procedures from the engineering viewpoint.

In the meanwhile, there are many smooth analytic approximations to the unit step function as it can be seen in the literature [4,5,6]. Besides, Sullivan et al [7] obtained a linear algebraic approximation to this function by means of a linear combination of exponential functions. However, the majority of all these approaches lead to closed - form representations consisting of non - elementary special functions, e.g. Gamma function, Hyperfunction, or Error function and also most of its algebraic exact forms are expressed in terms generalized integrals or infinitesimal terms, something that complicates the related computational procedures. In Ref. [8] an analytic exact form of the Unit Step Function was proposed as a summation of two inverse tangent functions. Nonetheless, according to this simplified approach the singularity structure was left ambiguous. Also, one may point out that a shortcoming of such formulae is that the involved inverse trigonometric functions do not have unique definitions.

Moreover, in Ref. [9] this special function this function was explicitly expressed by the aid of purely algebraic representations. The novelty of this work was that the proposed explicit formula is not performed in terms of non-elementary special functions, e.g. Error function.

Further, another elegant approximation to Heaviside function in the form of a summation of two logarithmic functions was carried out by Murphy in Ref. [10]. In this interesting work, the author also presented an explicitc form of Dirac delta function. 
In Ref. [11] the Unit Step Function was approached by some cumulative distribution functions (e.g. HalfCauchy and Hyperbolic-secant functions), whereas in Ref. [12] an analogous study was carried out towards the approximation of Unit Step Function by some other cumulative distribution functions.

Besides, in Ref. [13] a Hausdorff approximation of Heaviside Step function by means of several sigmoid functions (log-logistic, transmuted $\log$-logistic and generalized logistic functions) was taken into account and in this framework, upper and lower bounds for the Hausdorff distance were derived.

Finally in Ref. [14] a single - valued function was introduced, which was proved to be synonymous with the Unit Step Function. This formula consists of purely algebraic representations and does not contain either generalized integrals or any other infinitesimal quantities.

In the present study, in the sense of Ref. [8] an analytic exact form of the Unit Step Function has been proposed as a summation of four inverse tangent functions. Indeed, this formula constitutes a purely algebraic representation since it does not contain special functions, generalized integrals or any other infinitesimal quantities. In this context, this formula seems to be very practical and may have good prospects towards the computational procedures that concern the applications of Heaviside function in Operational Calculus, as well as in other engineering practices.

\section{Towards an exact form of Heaviside Function}

Let us introduce the following single - valued function $f: R^{*} \rightarrow R$ with

$$
f(x)=\frac{1}{\pi} \arctan \left(x^{n}\right)+\frac{2}{\pi} \arctan \left(\frac{x^{n}}{x^{2 n}+1}\right)+\frac{1}{\pi} \arctan \left(\frac{1}{x^{n}}\right)+\frac{2}{\pi} \arctan \left(\frac{x^{2 n}-x^{n}+1}{x^{2 n}+x^{n}+1}\right) \forall n \in N
$$

\section{Claim}

The function $f$ coincides with Heaviside Step Function over its domain of definition.

\section{Proof}

We shall prove that the value of the function $f$ vanishes for negative arguments and equals unity for strictly positive arguments.

To this end, let us calculate the first derivative of $f$ with respect to variable $x$.

For facility reasons, let us set $x^{n}=t$ with $t \in R^{*}$ and therefore

$\frac{d t}{d x}=n x^{n-1}$

Thus we can write out

$\frac{d}{d x} f(x)=\left[\frac{1}{t^{2}+1}+\frac{\frac{1}{t^{2}+1}-\frac{2 t^{2}}{\left(t^{2}+1\right)^{2}}}{\frac{t^{2}}{\left(t^{2}+1\right)^{2}}+1}-\frac{1}{t^{2}+1}+\frac{\frac{2 t-1}{t^{2}+t+1}-\frac{(2 t+1) \cdot\left(t^{2}-t+1\right)}{\left(t^{2}+t+1\right)^{2}}}{\frac{\left(t^{2}-t+1\right)^{2}}{\left(t^{2}+t+1\right)^{2}}+1}\right] \cdot n x^{n-1}$

After some algebra, it implies that

$\frac{d}{d x} f(x)=\left(\frac{t^{2}-1}{t^{4}-3 t^{2}+1}-\frac{t^{2}-1}{t^{4}-3 t^{2}+1}\right) \cdot n x^{n-1} \Leftrightarrow$ 
$\frac{d}{d x} f(x)=0, \forall x \in(-\infty, 0) \cup(0,+\infty)$

Here one may also pinpoint that since the left sided and right sided limits of the quantity: $\arctan \left(\frac{1}{t}\right)$, letting $t$ tend to zero (or equivalently $\arctan \left(\frac{1}{x^{n}}\right)$, letting $x$ tend to zero) are not equal, the limit of $f(x)$ letting $x$ tend to zero does not exist.

Next, let us focus on the set $(-\infty, 0)$ and suggestively estimate the value of $f(x)$ at $x=-1$

Thus we can write out

$$
\begin{aligned}
& f(-1)=\frac{1}{\pi} \arctan (-1)+\frac{2}{\pi} \arctan \left(-\frac{1}{2}\right)+\frac{1}{\pi} \arctan (-1)+\frac{2}{\pi} \arctan (3) \Rightarrow \\
& f(-1)=\frac{2}{\pi} \arctan (-1)+\frac{2}{\pi} \arctan \left(-\frac{1}{2}\right)+\frac{2}{\pi} \arctan (3)=0
\end{aligned}
$$

Since $\frac{d}{d x} f(x)=0, \forall x \in(-\infty, 0)$ and $f(-1)=0$ one may deduce that the value of $f(x)$ is zero for strictly negative arguments.

Then, let us focus on the set $(0,+\infty)$ and suggestively estimate the value of $f(x)$ at $x=1$

Thus we can write out

$$
\begin{aligned}
& f(1)=\frac{2}{\pi} \arctan (1)+\frac{2}{\pi} \arctan \left(\frac{1}{2}\right)+\frac{2}{\pi} \arctan \left(\frac{1}{3}\right) \Rightarrow \\
& f(1)=\frac{2}{\pi}\left(\arctan (1)+\arctan \left(\frac{1}{2}\right)+\arctan \left(\frac{1}{3}\right)\right) \Rightarrow \\
& f(1)=1
\end{aligned}
$$

Since $\frac{d}{d x} f(x)=0, \forall x \in(0,+\infty)$ and $f(1)=1$ one may infer that $f(x)$ equals unity for strictly positive arguments.

After all, it was proved that $f(x)$ is synonymous with Heaviside Step Function over its domain of definition.

\section{Discussion}

In the previous Section, we proposed an explicit form of Unit Step Function as a summation of four inverse tangent functions. However, one may pinpoint that a shortcoming of the single - valued function $f(x)$ introduced by eqn. (1) is that it cannot be defined at $x=0$. Besides, the limit of this function at $x=0$ does not exist, since the left sided and right sided limits of $f(x)$ letting $x$ tend to zero are not equal. 
Here, one may also remark that when Heaviside step function $H(x)$ is approached by the well - known logistic function $\frac{L}{1+E X P\left(-k\left(x-x_{0}\right)\right)}$, it is admitted beforehand that $H(0)=\frac{1}{2}$ [15].

In addition, one may also observe that a disadvantage of all mathematical formulae consisting of inverse trigonometric functions is that these functions do not have unique definitions. Moreover, one should elucidate that mathematical representations containing such quantities are not appropriate for determining the Dirac function by differentiating the proposed function with respect to variable $x$. For instance, it was shown that the first derivative of $f(x)$ with respect to $x$ vanishes over the $\operatorname{set}(-\infty, 0) \cup(0,+\infty)$. Further, one should emphasize that the formula introduced by eqn. (1) does not describe only one function but in fact describes a family of functions, that all have the same property (to be synonymous with Heaviside function) and this is an advantage over the mathematical formula proposed in Ref. [8].

Finally, on the basis of eqn. (1) one may also propose the following single - valued function expressed in power series form, which coincides as well with the Unit Step Function over the $\operatorname{set}(-\infty, 0) \cup(0,+\infty)$.

$g: R^{*} \rightarrow R$ with

$g(x)=\frac{1}{n \pi} \sum_{m=1}^{n}\left(\arctan \left(x^{m}\right)+2 \arctan \left(\frac{x^{m}}{x^{2 m}+1}\right)+\arctan \left(\frac{1}{x^{m}}\right)+2 \arctan \left(\frac{x^{2 m}-x^{m}+1}{x^{2 m}+x^{m}+1}\right)\right)$

where $m, n \in N^{*}$

\section{Conclusions}

The objective of this theoretical investigation was to introduce an analytic exact form of the Unit Step function. The proposed formula, constitutes a linear combination of four inverse trigonometric functions and therefore does not contain either generalized integrals or any other infinitesimal quantities. In addition, no other special functions are involved (e.g. Gamma function, Complementary Error function etc). In this famework, the proposed formula may have good prospects towards the computational procedures that concern the applications of the Unit Step function in Operational Calculus, as well as in many engineering practices. Nevertheless, one should pinpoint that a shortcoming of the proposed formula is that it cannot be defined at $x=0$.

\section{References}

[1] M. Abramowitz, I.A. and Stegun, Handbook of Mathematical Functions with Formulas, Graphs and Mathematical Tables, Dover Publications INC (1972).

[2] B.J. Hunt, Oliver Heaviside. Phys. Today, Vol. 65, Nuber 11, pp. 48-55

[3] R. Bracewell Heaviside's Unit Step Function, H(x). The Fourier Transform and its Applications, McGraw - Hill (2000).

[4] L. Berg Introduction to the Operational Calculus, North - Holland Publishing Company (1967).

[5] R.P. Kanwal, Generalized Functions Theory and Technique: Theory and Technique. 2nd ed. Boston, MA: Birkhäuser (1998)

[6] J. Spanier, J. K.B. and Oldham The Unit-Step u(x-a) and Related Functions Ch. 8 from: An Atlas of Functions. Washington, DC: Hemisphere, pp. 63-69, 1987

[7] J. Sullivan, L. Crone and J. Jalickee, Approximation of the Unit Step Function by a Linear Combination of Exponential Functions, Journal of. Approximation Theory, Vol. 28, Number 3, pp. 299-308, 1980

[8] J. Venetis, An analytic exact form of the unit step function, Mathematics and Statistics Vol. 2, Number 7, pp. 235-237, 2014 
[9] J. Venetis, An analytic exact form of the Heaviside step function Advances and Applications in Discrete Mathematics, Volume 22, Number 2, 2019, Pages 153-159

[10] M. Kyle Murphy, Explicit Forms of Discontinuous Functions the Dirac Delta and Irreducible Forms (preprint) https://www.academia.edu/11704122/Explicit_Forms_of_Discontinuous_Functions_the_Dirac_Delta_and_Irreduci ble_Forms

[11] N. Kyurkchiev, On the Approximation of the step function by some cumulative distribution functions. Compt. rend. Acad. bulg. Sci. Vol. 68, Number 4, pp. 1475-1482, 2015

[12] V. Kyurkchiev, N. Kyurkchiev, On the Approximation of the Step function by Raised - Cosine and Laplace Cumulative Distribution Functions, European International Journal of Science and Technology Volume 4, Number 2, pp. 75-84, 2016.

[13] A. Iliev, N. Kyurkchiev, S. Markov, On the approximation of the step function by some sigmoid functions, Mathematics and Computers in Simulation, Volume 133, Number 1, pp. 223-234, 2017

[14] J. Venetis, Analytic Exact Forms of Heaviside and Dirac Delta Function, Advances in Dynamical Systems and Applications, Vol. 15, Number 1, pp. 115-121, 2020

[15] I. A. Maron, Problems in Calculus of One Variable, with Elements of Theory, Translated from the Russian by Leonid Levant [Revised from the 1970 Russian Ed.] Mir Publishers, 1973 